\section{Acute lymphoblastic leukemia: age and biology}

\author{
Robin Foà \\ Division of Hematology, Department \\ of Cellular Biotechnologies and Hematology, \\ Sapienza University, Rome, Italy
}

\section{Abstract}

Acute lymphoblastic leukemia (ALL) is the most frequent neoplasm in children, while being relatively rare in adults. The outcome of children with ALL is far superior than that observed in adults, whose survival rates generally do not exceed $40 \%$. A retrospective analysis recently carried out on a large series of cases enrolled in the AIEOP and GIMEMA protocols for the treatment of pediatric and adult ALL has documented specific differences among the various age cohorts examined, particularly in terms of incidence of molecular rearrangements, with the $B C R / A B L$ rearrangement being detected in more than half of patients in the $6^{\text {th }}$ decade of life. These findings highlight the importance of a precise diagnostic screening at all ages, since elderly patients might benefit more from targeted approaches, that are associated with less toxic effects. Furthermore, extended biologic approaches aimed at identifying novel therapeutic targets should be regarded as a main goal to refine our therapeutic armamentarium.

Finally, the introduction of pediatric-like protocols is progressively changing the outcome of young adult patients, although an important caveat is represented by the comorbidities and toxic effects associated with more aggressive chemotherapy; therefore, patients' fitness should always be carefully considered.

Acute lymphoblastic leukemia (ALL) is the most frequent neoplasm in children, whereas it is more rare in adults. Over the last decades, there has been a considerable improvement in the management and outcome of childhood ALL, with CR rates and long-term survivals reaching 95\% and $80 \%$, respectively. At variance, in adults the clinical scenario is significantly different, mostly in terms of survival rates, which generally do not exceed $40 \%$.

Several features can at least in part explain this marked difference in prognosis; these include - among others - more intensive regimens, less side effects and a higher compliance to aggressive chemotherapy schedules in children, while in adults the toxic effects often represent a considerable limit to the administration of adequate drug doses and to the correct timing of chemotherapy. As a proof of principle of these concepts, it has been widely reported that adolescents and young adults (also called AYA) treated with pediatric-like regimens, have a superior outcome.

Moreover, a number of biologic features are responsible for the different clinical scenario, such as an increased incidence in older patients of the $B C R / A B L$ and ALL1/AF4 transcripts, known to have a negative impact on prognosis, and a decreased incidence of the ETV6/RUNX1 rearrangement, associated with a favorable outcome; furthermore, it has been reported that older patients ( $>60$ years) tend to have a decreased male/female ratio and a decreased incidence of organomegaly.

Taking advantage of the availability of two large databases of the Italian multicenter protocols AIEOP (Associazione Italiana Ematologia ed Oncologia Pediatrica) and GIMEMA (Gruppo Italiano Malattie EMatologiche dell'Adulto) for the treatment of pediatric and adult ALL, respectively, we have recently evaluated retrospectively the clinicobiologic features of more than 5000 patients, subdivided in different age groups (1-5, 5-10, 10-14, 14-18, 18-25, 25-20, 30-40, 40-50, 50-60 years).

The analysis of this large cohort of patients has allowed to highlight, as expected, a peak in the incidence of ALL in the age groups 1-5 and 5-10, with a progressive decrease from 10 to 30 years and an increase from 30 years onwards. Interestingly, gender distribution highlighted an overall lower incidence of females: this phenomenon was remarkable between $14-50$ years and disappeared in the 50-60 age-cohort. Furthermore, there was a significant association between gender and immunophenotype, T-ALL being more frequent in males up to the $4^{\text {th }}$ decade. Molecular screening showed that the ETV6/RUNX1+ virtually disappeared in adult patients; furthermore, it also highlighted a progressive increase of two molecular rearrangements known to be associated with a very poor outcome, namely ALL1/AF4 and $B C R / A B L$, the latter being detected in more than $50 \%$ of individuals in the $6^{\text {th }}$ decade of life.

These findings provide a further biologic rationale for the progressive implementation of novel targeted therapeutic approaches directed against specific targets. Two illuminating examples in this sense are given by two consecutive Italian protocols for the treatment of adult $B C R / A B L+$ ALL patients. The first protocol, the GIMEMA LAL 0201B study, enrolled only elderly patients ( $>60$ years) and was based on the administration of corticosteroids for the first week of treatment followed thereafter by imatinib, the first tyrosine kinase inhibitor specifically directed against the ABL protein, at the dose of $800 \mathrm{mg}$ daily, associated to steroids without further chemotherapy as frontline treatment. The median age was 69 years and, remarkably, all patients obtained a complete hematologic remission, with a consistent reduction of the BCR-ABL transcripts. This schedule allowed a reduced hospitalization, was not coupled to major toxicities and treatment was well tolerated.

In the second study, the GIMEMA LAL1205 protocol, designed for adult individuals ( $>18$ years)
Correspondence: Robin Foà, Division of Hematology, Department of Cellular Biotechnologies and Hematology, Sapienza University of Rome, Via Benevento 6, 00161 Rome, Italy.

Tel: +39-06-85795753 - Fax: +39-06-85795792.

E-mail: rfoa@bce.uniromal.it

Key words: acute lymphoblastic leukemia, biology.

Received for publication: 4 May 2011.

Accepted for publication: 4 June 2011.

This work is licensed under a Creative Commons Attribution NonCommercial 3.0 License (CC BYNC 3.0).

(C) Copyright R. Foà, 2011

Licensee PAGEPress, Italy

Pediatric Reports 2011; 3(s2):e2

doi:10.4081/pr.2011.s2.e2

with a BCR/ABL+ ALL, dasatinib - one of the most potent second-generation tyrosine kinase inhibitors - was administered $70 \mathrm{mg}$ twice daily for a total of 84 days, and was preceded by a week of corticosteroids: also with this schedule, all patients obtained a complete hematologic remission and had a good quality of life.

Overall, these two studies have shown that also elderly patients with $B C R / A B L+$ ALL, previously considered eligible only for palliative treatment strategies, may be spared chemotherapy and successfully managed with a targeted therapeutic approach. Furthermore, they underline the importance of performing a precise diagnostic work-up at all ages, with the final goal of providing all patients with the best therapeutic option.

Another example is represented by the specific overexpression of the Flt3 kinase in patients carrying the ALL1/AF4 rearrangement and in a subgroup of children and adults without known molecular aberrations. Although this finding has so far not translated into the clinical practice, it is intriguing to speculate that regimes including Flt3 inhibitors might be of benefit for ALL patients who have high expression levels of this kinase and who are known to have an unfavorable prognostic likelihood.

In conclusion, stratification of patients according to age groups is associated with important clinical and biologic features that might be in part responsible of the marked differences in the outcome witnessed in children and adults with ALL.

The introduction of pediatric-like regimes for the management of young adults is progressively changing the outcome for this subgroup of patients, although some important questions still remain open. In particular, a clear cut-off for the definition of young adults has so far not been established and the burden of toxicities associated with these more aggressive therapeutic regimens in terms of compliance to treatment, quality of life and overall survival is at present not conclusively defined for the different age ranges. 